\title{
WEAK COMPACTNESS IN AN OPERATOR SPACE
}

\section{By Hisaharu UMEgaKI}

1. Introduction. Many important theorems in measure theory have been extended to $W^{*}$-algebras by many authors, especially, Dixmier [2], Dye [3] and Segal [8]. Considered as non-commutative extensions, these extensions are interesting themselves and provide powerful tools in the further investigations of $W^{*}$-algebra. In the previous papers ([11] and [12]), we have discussed and extended the concepts of conditional expectations, which have been introduced by Dixmier in the operator theoretical term [2], and martingales in the probability theory into finite and semi-finite $W^{*}$-algebras. The concept of the former has been also discussed in a general situation by Nakamura-Turumaru [6].

The purpose of the present paper is to extend certain compactness theorems in $L^{\prime}$ on measure space to $L^{\prime}$ on $W^{*}$-algebra in the sense of [8] and [2]. Firstly, as a preliminary we shall prove the extension of Vitali-Hahn-Saks's Theorem for any $W^{*}$-algebra $A$ with a regular gage $\mu(\mathrm{cf} . \S 3)$ (that is, a regular gage space $(A, \mu)$ in the sense of [8]), which implies the equi-absolute continuities of weakly convergent sequence in $L^{\prime}(A, \mu)$. Secondly, we shall extend the Lebesgue's compactness theorem to $W^{*}$-algebra with respect to a finite gage and give a sufficient condition for a subset in $\left(A_{*}\right)^{+}$to be weakly compact $\left(A_{*}\right.$ being a Banach space in the notation of [2], cf. $\S 4$ as below). The former characterizes the weakly conditional compactness of a subset in $L^{\prime}(A)$, and the latter is possible to extend a Kakutani's compactness theorem in $L^{\prime}$ (with respect to measure space) to the present $L^{\prime}(A)$ with respect to arbitrary gage (cf. $\S 4)$. In the last part of $\S 4$, we shall also characterize the weakly sequential compactness of subset in $L^{\prime}(A, \mu)^{+}$by a uniform continuity of the set in the form of Bartle-Dunford-Schwartz [1], and further prove weakly sequential completeness of $L^{\prime}(A, \mu)$ for $A$ of finite type and any gage $\mu$.

2. Preliminary and notations. Let " $\mathfrak{P}$ " be the set of all projections in the $W^{*}$-algebra $A$ acting on a Hilbert space $H$. For any $p \in \mathfrak{P}$ there corresponds uniquely a closed linear subspace $\mathfrak{M}_{p} \subset H$ such that the projection from $H$ onto $\mathfrak{M}_{p}$ coincides with $p$. For any $p, q \in \mathfrak{P}$, the meet $p \wedge q$ and the join $p \vee q$ are uniquely defined as the projections onto $\mathfrak{M}_{p} \cap \mathfrak{M}_{q}$ and $\mathfrak{M}_{p} \oplus \mathfrak{M}_{q}$ respectively. Whence $\mathfrak{B}$ is a complete lattice with respect to the $\wedge$ and $\vee$.

Let $\mu$ be a gage of $A$ in the sense of [8], i. e. non-negative valued, unitary

Received November 1, 1956. 
invariant and completely additive function on $\mathfrak{P}$ satisfying that every $p \in \mathfrak{P}$ is 1 . u. b. of $\mu$-finite projections $q \in \mathfrak{P}$ within $q \leqq p$. Denote by $P_{\mu}$ (or merely $P$ ) the set of all $\mu$-finite projections in $\mathfrak{P}$. A gage $\mu$ of $A$ is called to be regular if it is faithful (cf. [8]), which coincides with the contraction onto $\mathfrak{P}$ of the "normale, fidèle, éssentielle et maximale" trace in the sense of [2]. Let $L^{\prime}(A, \mu)$ and $L^{2}(A, \mu)$ (or merely $L^{\prime}(A)$ and $L^{2}(A)$ ) be the space of all integrable or square integrable operators with respect to a fixed gage $\mu$ with the norms $x{ }_{1}$ and $\left.x\right|_{2}$ respectively. The gage $\mu$ is uniquely extended to a positive linear functional on $L^{\prime}(A)$, which is also denoted by $\mu(x)$ $\left(x \in L^{\prime}(A)\right)$.

For any $W^{*}$-subalgebra $B_{1}$ of $A$, let $I_{0}=1$.u. b. $\left\{p ; p \in P \cap B_{1}\right\}$ which belongs to the center of $B_{1}$ and $I_{0} B_{1}\left(=B\right.$, say) is considered as a $W^{*}$-algebra on the Hilbert space $I_{0} H$. The contracted function of $\mu$ onto $\mathfrak{P} \cap B$ (denote it by the same notation $\mu)$ is also a gage of $B$ and $L^{\prime}(B, \mu)$ is a subspace of $L^{\prime}(A, \mu)$, which is uniquely determined by $\left(B_{1}, \mu\right)$. We denote it by $L^{\prime}\left(B_{1}, \mu\right)$. If $\mu$ is regular on $A$, then it is also regular on $B$.

Denote the set of all non-negative operators in $L^{\prime}(A)$ by $L^{\prime}(A)^{+}$. Any $x \in$ $L^{\prime}(A)$ is uniquely expressed by $x=x^{(1)}-x^{(2)}+i x^{(3)}-i x^{(4)}$ with $x^{(j)} \in L^{\prime}(A)^{+}$. Put $x^{\prime}=x^{(1)}-x^{(2)}$ and $x^{\prime \prime}=x^{(3)}-x^{(4)}$, which are real and imaginary parts of $x$ respectively.

For any $x \in L^{\prime}(A), W(x)$ denotes the $W^{*}$-subalgebra generated by $\left\{e_{\lambda}\left(x^{\prime}\right)\right.$, $\left.e_{\lambda}\left(x^{\prime \prime}\right)\right\}_{\lambda}$ where $x^{\prime}=\int \lambda d e_{\lambda}\left(x^{\prime}\right)$ and $x^{\prime \prime}=\int \lambda d e_{\lambda}\left(x^{\prime \prime}\right)$. Further for any subset $S$ in $L^{\prime}(A)$, " $W(S)$ " denotes the $W^{*}$-subalgebra generated by $\{W(x) ; x \in S\}$.

If $E$ is a Banach space, $E^{\wedge}$ denotes the conjugate space of $E$. The weak topology in $E$ as point is merely called by weak topology or $\sigma\left(E, E^{\wedge}\right)$-topology, and the weak topology in $E^{\wedge}$ as functional is called weak* topology or $\sigma\left(E^{\wedge}, E\right)$-topology. The conjugate space of $L^{\prime}(A)$ is denoted by $L^{\infty}(A)$.

3. Equi-absolute continuity of a convergent sequence of functionals. Firstly, we give a fundamental definition:

Definition 1. Let $A$ be a $W^{*}$-algebra with a gage $\mu$. A set $S$ of linear functionals on $A$ is called to be equi $\mu$-absolutely continuous, if for any real $\varepsilon>0$ there exists a real $\delta>0$ such that

$$
\mu(p)<\delta(p \in \mathfrak{P}) \text { implies }|f(p)|<\varepsilon \text { for all } f \in S \text {. }
$$

Similarly if $S$ is a subset of $L^{\prime}(A, \mu)$ and $\left\{f_{x} ; x \in S\right\}$ (where $f_{x}(y)=\mu(x y)$ for all $y \in A$ ) satisfies (1), then $S$ is called to be equi $\mu$-absolutely continuous.

For any given semi-finite $W^{*}$-algebra $A$ acting on a Hilbert space $H$ and a regular gage $\mu$ of $A$ (it is known by Dixmier that such $A$ has always regular gage), Vitali-Hahn-Saks's Theorem can be extended to this $(A, \mu)$ :

Theorem 1. Let $\left\{f_{n}\right\}$ be a sequence of linear functionals on $A$ which are strongly continuous on the unit sphere of $A$. If for every projection $p$ in $A$ $\lim _{n \rightarrow \infty} f_{n}(p)$ exists and is finite, then the set $\left\{f_{n}\right\}$ is equi $\mu$-absolutely continuous. 
LemMa 1.1. For any pair $p, q \in P$, putting $\rho(p, q)=\sqrt{\mu\left(p-\left.q\right|^{2}\right), \rho \text { satisfies }}$ the metric conditions and $(P, \rho)$ is a complete metric space.

Proof. It follows from [2] or [8] that $\rho$ satisfies the metric conditions. Now let us prove the completeness of $(P, \rho)$. Taking $\left\{p_{n}\right\} \subset P$ such that $\rho\left(p_{m}, p_{n}\right) \rightarrow 0$ (as $\left.m, n \rightarrow \infty\right)$, by the completeness of $L^{2}(A, \mu)$, there exists an $x \in L^{2}(A, \mu)$ such that $\mu\left(x-\left.p_{n}\right|^{2}\right) \rightarrow 0$ (as $\left.n \rightarrow \infty\right)$. Since $0 \leqq p_{n} \leqq 1, p_{n}$ converges strongly to $x$ on the Hilbert space $H$ and $0 \leqq x \leqq 1$ on $H$. Hence for any $\xi, \eta \in H$

$$
(x \xi, \eta)=\lim _{n \rightarrow \infty}\left(p_{n} \xi, \eta\right)=\lim _{n \rightarrow \infty}\left(p_{n} \xi, p_{n} \eta\right)=(x \xi, x \eta)=\left(x^{2} \xi, \eta\right) .
$$

Since $x^{*}=x$ in $L^{2}(A, \mu)$, we get $\mu(x)<\infty$ and $x \in P$.

Lemma 1.2. For any $\delta>0$ and $p_{0} \in P$, putting $U_{\delta}\left(p_{0}\right)=\left\{p \in P ; \rho\left(p_{0}, p\right)\right.$ $<\sqrt{ } \delta\}$ and $V_{\delta}\left(p_{0}\right)=\left\{p \in P ; \mu(p)<\mu\left(p_{0}\right)+\delta, \mu\left(p p_{0}\right)>\mu\left(p_{0}\right)-\delta\right\}$, then $V_{\delta}\left(p_{0}\right) \subset U_{3 \delta}\left(p_{0}\right)$.

Proof. If $p \in V_{\delta}\left(p_{0}\right)$, then

$$
\begin{aligned}
\rho\left(p_{0}, p\right)^{2} & =\mu\left(p_{0}-\left.p\right|^{2}\right)=\mu\left(\left(p_{0}-p\right)^{2}\right)=\mu\left(p_{0}\right)+\mu(p)-2 \mu\left(p p_{0}\right) \\
& <2 \mu\left(p_{0}\right)+\delta-2 \mu\left(p_{0}\right)+2 \delta=3 \delta .
\end{aligned}
$$

Therefore $p \in U_{3 \delta}\left(p_{0}\right)$.

Proof of Theorem 1. Since each $f_{n}$ is a continuous function on $(P, \rho)$, for any fixed integer $n_{0}>0$ and any fixed $\varepsilon>0$ putting

$$
E_{n_{0}}=\left\{p \in P ; \sup _{m, n \geqq n_{0}}\left|f_{m}(p)-f_{n}(p)\right| \leqq \varepsilon / 4\right\},
$$

each $E_{n_{0}}$ is closed in $(P, \rho)$ and $\bigcup_{n_{0}=1}^{\infty} E_{n_{0}}=P$ by the assumption of $\left\{f_{n}\right\}$. By Lemma 1.1 and Baire's category theorem, for some $n_{0} E_{\imath_{0}}$ has a non-empty interior in $(P, \rho)$. Therefore there exist $p(\neq 0) \in P$ and $\delta>0$ such that $U_{3 \delta}(p)$ is non-empty and contained in $E_{n_{0}}$. Let $q$ be any fixed projection in $P$ with $\mu(q)<\delta_{1}=\min (\delta, \mu(p))$. Putting $r=p \vee q$, we have

$$
\mu(r-p) \leqq \mu(p)+\mu(q)-\mu(p)=\mu(q)<\delta
$$

and $\mu(r p)=\mu(p)>\mu(p)-\delta$, i. e. $r \in V_{\delta}(p)$. Furthermore, since $\mu(q)$ $<\mu(p)$, we get $r>q, \mu(r-q) \leqq \mu(p)<\mu(p)+\delta$ and

$$
\mu((r-q) p)=\mu(p)-\mu(q p)>\mu(p)-\delta,
$$

i. e. $r-q \in V_{\delta}(p)$. Hence we deduce that $r, r-q \in E_{n_{0}}$. Since $q=r-(r$ $-q)$

$$
\begin{aligned}
& f_{m}(q)-f_{n}(q) \leqq f_{m}(\boldsymbol{r})-f_{n}(\boldsymbol{r}) !+f_{m}(\boldsymbol{r}-q)-f_{n}(\boldsymbol{r}-q) \\
& <\varepsilon / 2 \quad \text { for all } m, n \geqq n_{0} \text {. }
\end{aligned}
$$

For $n=1,2, \cdots, n_{0}$, we can find a $\delta_{2}>0\left(\delta_{2}<\delta_{1}\right)$ such that

$$
f_{n}(q) \mid<\varepsilon / 2 \quad \text { for any } q \in P \text { with } \mu(q)<\delta_{2}
$$


and $n=1,2, \cdots, n_{0}$. Consequently we obtain that $\mu(q)<\delta_{2}$ implies

$$
\left|f_{n}(q)\right| \leqq\left|f_{n}(q)-f_{n_{0}}(q)\right|+\left|f_{n_{0}}(q)\right|<\varepsilon \quad \text { for all } n \geqq n_{0} .
$$

(2) and (3) imply the equi $\mu$-absolute continuity of $\left\{f_{n}\right\}$.

REMARK 1. The above proof has done under the Lemmas 1.1 and 1.2 by a similar proof of classical Vitali-Hahn-Saks's Theorem ${ }^{1)}$, in which the metric $\rho$ is defined (denote it $\rho_{1}$ as the following) by the $L^{\prime}$-norm, i.e. $\rho_{1}(p, q)=\mu(p$ $-q$ ) for $p, q \in P$. If the gage $\mu$ is finite, then the metrics $\rho$ and $\rho_{1}$ are equivalent, and the neighborhood topologies in $P$ defined by $\left\{U_{\delta}(p)\right\}$ and $\left\{V_{\delta}(p)\right\}$ (cf. Lemma 1.2) are also equivalent to the metric topology.

4. Weak compactness of subset in $L^{\prime}(A, \mu)$. A subset $K$ of a Banach space $E$ is called to be weakly (or equally $\sigma\left(E, E^{\wedge}\right)$-) conditionally compact, if the weak $\left(\sigma\left(E, E^{\wedge}\right)-\right)$ closure of $K$ is weakly $\left(\sigma\left(E, E^{\wedge}\right)-\right)$ compact subset of $\left.E_{0}{ }^{2}\right)$ Firstly we shall extend Lebesgue's compactness theorem to a $W^{*}$-algebra $A$.

THeOREM 2. Let $\mu$ be a gage of $A$ with $\mu(I)<\infty$. Then, for a subset $K$ of $L^{\prime}(A, \mu)$ to be weakly conditionally compact it is necessary and sufficient that $K$ is equi $\mu$-absolutely continuous and $K^{\prime}, K^{\prime \prime}$ are bounded in the $L^{\prime}$-norm where $K^{\prime}=\left\{x^{\prime} ; x \in K\right\}$ and $K^{\prime \prime}=\left\{x^{\prime \prime} ; x \in K\right\}$.

Lemma 2.1. For any equi $\mu$-absolutely continuous subset $K$ of $L^{\prime}(A, \mu), K$, $(j=1, \cdots, 4)$ are also equi $\mu$-absolutely continuous, where $K_{j}=\left\{x^{(j)} ; x \in K\right\}$.

Proof. ${ }^{3)}$ Since for every projection $p$

$$
|\mu(x p)|^{2}=\left|\mu\left(x^{\prime} p\right)\right|^{2}+\left|\mu\left(x^{\prime \prime} p\right)\right|^{2},
$$

$K^{\prime}$ and $K^{\prime \prime}$ are equi $\mu$-absolutely continuous. For fixed $x^{\prime}$, there exists $q \in \mathfrak{P}$ such that $x^{(1)}=q x^{\prime}=x^{\prime} q$ and $x^{(2)}=(1-q) x^{\prime}=x^{\prime}(1-q)$. For any $\varepsilon>0$ and $K^{\prime}$, take $\delta>0$ as in (1). Since $\mu(p)<\delta(p \in \mathfrak{P})$ implies $\mu(q p q)<\delta$ and $\mu((1-q) p(1-q))<\delta$,

$$
0 \leqq \mu\left(p x^{(1)}\right)=\mu\left(p q x^{\prime}\right)=\mu\left(q p q x^{\prime}\right)<\varepsilon
$$

and similarly $0 \leqq \mu\left(p x^{(2)}\right)<\varepsilon$. Hence $K_{1}$ and $K_{2}$ are equi $\mu$-absolutely continuous, and also similarly for $K_{3}$ and $K_{4}$.

Proof of Theorem 2. (Sufficiency). Let $\bar{K}_{i}(j=1, \cdots, 4)$ be $\sigma\left(L^{\infty}, L^{\infty \wedge}\right)$-closures of $K_{j}$ respectively which are $\sigma\left(L^{\infty}, L^{\infty \wedge}\right)$-compact in $L^{\infty \wedge}$. For any $p \in \mathfrak{B}$ with $\mu(p)<\delta$ and for any fixed $f \in \bar{K}_{1}$ there exists $x \in K$ such that

$$
\left|f(p)-\mu\left(x^{(1)} p\right)\right|<\varepsilon .
$$

1) See Saks [7] for finite measure space and also see e.g. Sunouchi [10] for $\sigma$ finite measure space.

2) Further, a subset $K$ of $E$ is called to be weakly (or equally $\sigma\left(E, E^{\wedge}\right)-$ ) sequentially conditionally compact, if any countable subset $C$ of $K$ contains always a sequence $\left\{x_{n}\right\}$ which converges weakly to some $x \in E$.

3) This proof also holds for any gage without finiteness $\mu(I)<\infty$. 
Since $0 \leqq \mu\left(x^{(1)} p\right)<\varepsilon$ for every such $p$,

$$
0 \leqq f(p) \leqq\left|f(p)-\mu\left(x^{(1)} p\right)\right|+\mu\left(x^{(1)} p\right)<2 \varepsilon,
$$

i. e. $0 \leqq f(p)<2 \varepsilon$ for every $p \in \mathfrak{P}$ with $\mu(p)<\delta$. Therefore by RadonNikodym's Theorem of Dye [3] there exists $z \in L^{\prime}(A)$ such that $f(y)=\mu(z y)$ for every $y \in A$. This means that $K_{1}$ is weakly conditionally compact in $L^{\prime}(A, \mu)$. Similarly we get $K_{j}(j=2,3,4)$. Consequently $K$ is weakly conditionally compact in $L^{\prime}(A, \mu)$.

(Necessity). For this purpose we can assume $\mu$ to be regular without loss of generality, and hence $A$ is countably decomposable and of finite type, because $\mu(I)<\infty$. Since $K^{\prime}$ and $K^{\prime \prime}$ are weakly sequentially conditionally compact (cf. [9]), they are bounded in the $L^{\prime}$-norm. Assuming the contrary of the equi $\mu$-absolute continuity of $K$, there exist $\left\{p_{n}\right\} \subset \mathfrak{P}$ and a weakly convergent sequence $\left\{x_{n}\right\} \subset K$ such that

$$
\mu\left(p_{n}\right)<\frac{1}{n} \text { and }\left|\mu\left(x_{n} p_{n}\right)\right|>\varepsilon
$$

for some $\varepsilon>0$ and for all $n=1,2, \cdots$. Putting $f_{n}(y)=\mu\left(x_{n} y\right)$ for $y \in A$, $\lim _{n \rightarrow \infty} f_{n}(y)$ exists for every $y \in A$ which contradicts (4) by Theorem 1 .

In a general situation, we can give a sufficient condition for weak compactness: Let $A$ be a $W^{*}$-algebra and $A_{*}$ be the Banach space of all linear functionals on $A$ which are strongly continuous on the unit sphere of $A$. Then $\left(A_{*}\right)^{\wedge}=A$ (cf. [2]). Denote the set of all non-negative functionals in $A_{*}$ by $\left(A_{*}\right)^{+}$, then

Corollary 2.1. If a subset $K$ of $\left(A_{*}\right)^{+}$is bounded in the norm of $A_{*}$ and satisfies

(5) for any decreasing directed set $\left\{p_{\alpha}\right\}$ of projections in A with $p_{\alpha} \downarrow 0, f\left(p_{a}\right)$ converges to 0 uniformly for every $f \in K$,

then $K$ is $\sigma\left(A_{*}, A\right)$-conditionally compact.

Proof. Since any completely additive positive linear functional on $A$ belongs to $A_{*}$ by Dixmier (cf. Théorème 1 and footnote 6 of [2]), the proof will be obtained by the method almost similar with the proof of suficiency of Theorem 2, that is, let $K$ being $\sigma\left(A, A^{\wedge}\right)$-closure of $K$, then every $f \in \bar{K}$ is non-negative linear functional on $A$, and by (5) $f$ is completely additive. Hence by the theorem of Dixmier $f$ belongs to $\left(A_{*}\right)^{+}$, and $K$ is $\sigma\left(A_{*}, A\right)$-conditionally compact.

By Corollary 2.1, Kakutani's compactness Theorem (cf. Theorem 10 of [5]) will be extended to the following:

Corollary 2.2. Let $A$ be a $W^{*}$-algebra with gage $\mu$. Let $x_{1}, x_{2} \in L^{\prime}(A, \mu)^{+}$ with $x_{1}<x_{2}$. Then $\left\{x ; x_{1} \leqq x \leqq x_{2}\right\}$ is weakly conditionally compact in $L^{\prime}(A, \mu)^{+}$.

Uuder the same notation of the above Corollary 2.2, we prove the following: 
Theorem 3. For a subset $K \subset L^{\prime}(A, \mu)^{+}$to be weakly sequentially conditionally compact, it is necessary and sufficient that $K$ is bounded in $L^{\prime}$-norm and satisfies

$\left(5^{\prime}\right)$ for any sequence of projections $\left\{p_{n}\right\}$ in $A$ with $p_{n} \downarrow 0, \mu\left(x p_{n}\right)$ converges to 0 uniformly for every $x \in K$.

Proof of sufficiency. In this case we can also assume $\mu$ to be regular without loss of generality. Let $\left\{x_{n}\right\} \subset K$. Putting $B_{1}=W\left(\left\{x_{n}\right\}\right)$ and $B=$ weak closure of $B_{1} \cap L^{\prime}(A, \mu), B$ is a countably decomposable $W^{*}$-algebra on a closed linear subspace of $L^{2}(A, \mu)$. Further $\left\{x_{n}\right\}$ is contained in $L^{\prime}\left(B_{1}, \mu\right)$ and satisfies $\left(5^{\prime}\right)$ on $(B, \mu)$. Therefore by Corollary $2.1,\left\{x_{n}\right\}$ is weakly conditionally compact in $L^{\prime}\left(B_{1}, \mu\right)$, and there exists a subsequence $\left\{x_{n_{k}}\right\} \subset\left\{x_{n}\right\}$ which converges weakly to an $x \in L^{\prime}\left(B_{1}, \mu\right)$, i. e. $\mu(x y)=\lim _{k} \mu\left(x_{n_{k}} y\right)$ for all $y \in B$. Let $y^{e}$ be the conditional expectation of $y \in A$ relative to $B_{1},{ }^{4)}$ then $\mu\left(z y^{e}\right)$ $=\mu(z y)$ for all $z \in B_{1} \cap L^{\prime}(A, \mu)$ (cf. [2] or [12]). Since $L^{\prime}\left(B_{1}, \mu\right)$ coincides with the $L^{\prime}(\mu)$-closure of $B_{1} \cap L^{\prime}(A, \mu), \mu\left(z y^{e}\right)=\mu(z y)$ for all $z \in L^{\prime}\left(B_{1}, \mu\right)$. Moreover $x$ and $x_{n}(n=1,2, \cdots)$ belong to $L^{\prime}\left(B_{1}, \mu\right)$ and therefore for every $y \in A$

$$
\mu(x y)=\mu\left(x y^{e}\right)=\lim _{k \rightarrow \infty} \mu\left(x_{n_{k}} y^{e}\right)=\lim _{k \rightarrow \infty} \mu\left(x_{n_{k}} y\right),
$$

that is, $x_{n_{k}}$ converges weakly to $x$ in $L^{\prime}(A, \mu)$ and $K$ is weakly sequentially conditionally compact.

Proof of necessity. The boundedness of $K$ in the $L^{\prime}$-norm is obvious. Assuming the contrary of $\left(5^{\prime}\right)$, there exist $\varepsilon_{1}>0,\left\{p_{n}\right\} \subset \mathfrak{P}$ and weakly convergent sequence $\left\{x_{n}\right\} \subset K$ such that

$$
p_{n} \downarrow 0 \text { and } \mu\left(x_{n} p_{n}\right)>\varepsilon_{1} \quad \text { for all } n=1,2, \cdots .
$$

Putting $f_{n}(y)=\mu\left(x_{n} y\right)(n=1,2, \cdots)$ and $\nu(y)=\sum_{n=1}^{\infty} f_{n}(y) / c \cdot 2^{n}\left(c=\sup \left\{x_{i 1}\right.\right.$; $x \in K\}), f_{n}$ are absolutely continuous with respect to $\nu$. Let $C$ be $W^{*}$-subalgebra generated by $\left\{p_{n}\right\}$ which is commutative. Hence by Vitali-Hahn-Saks's Theorem on commutative case of Theorem 1 or on usual measure space, for any $\varepsilon>0$ there exists $\delta>0$ such that $f_{n}(p) \mid<\varepsilon$ for every $p \in \mathfrak{P} \cap C$ with $\nu(p)<\delta$. Since $\nu\left(p_{n}\right) \rightarrow 0($ as $n \rightarrow \infty), \mu\left(x_{n} p_{n}\right)=f_{n}\left(p_{n}\right) \rightarrow 0$, (7) yields a contradiction.

REMARK 2. This theorem has been proved by Bartle-Dunford-Schwartz (cf. Theorem 1 of [1]) for subset of space of measures on abstract set and the proof of necessity is done by a method similar with that. If $A$ is commutative, then we get a similar fact with [1], i. e. taking a gage $\mu$ of $A$, for subset $K$ of $L^{\prime}(A, \mu)$ without the restriction that $K \subset L^{\prime}(A, \mu)^{+}$, Theorem 3 will be obtained by our proof, because any countably additive linear functional on the $W^{*}$-algebra $B$ (cf. proof of Theorem 3) is strongly continuous on its unit sphere. We have also the same fact for subset $K$ in $A_{*}$, because $A_{*}$ is isometrically isomorphic to $L^{\prime}(A, \mu)$ with respect to a regular gage $\mu$ on $A$.

4) The notion of the conditional expectation refers to [12]. 
REMARK 3. In Theorem 3, applying the Eberlein's Theorem (cf. [4]), if $K$ is weakly closed, then the condition is necessary and sufficient for $K$ to be weakly compact.

Applying Theorem 2 and the proof of Theorem 3, we have

Corollary 3.1. Let $A$ be a finite $W^{*}$-algebra and let $\mu$ be any fixed gage. Then $L^{\prime}(A, \mu)$ is weakly sequentially complete.

Proof. Again we can assume $\mu$ to be regular. Let $\left\{x_{n}\right\} \subset L^{\prime}(A, \mu)$ be a sequence with finite $\lim \mu\left(x_{n} y\right)$ for all $y \in A$. For this $\left\{x_{n}\right\}$, we take the $W^{*}$-algebras $B_{1}$ and $B$ as in the proof of Theorem 3. Then $B$ has a finite regular gage $\tau$. Putting $f_{n}(y)=\mu\left(x_{n} y\right)$ for $y \in A, f_{n}$ are strongly continuous on the unit sphere of $B$ and there exists $z_{n} \in L^{\prime}(B, \tau)$ such that $f_{n}(y)=\tau\left(z_{n} y\right)$ for all $y \in B$ and $n=1,2, \cdots$. Since $\lim f_{n}(y)(=f(y)$ say) exists and is finite for every $y \in A,\left\{z_{n}{ }^{\prime}\right\}$ and $\left\{z_{n}{ }^{\prime \prime}\right\}$ are bounded in $L^{\prime}(\tau)$-norm and by Theorem 1 $\left\{z_{n}\right\}$ is equi $\tau$-absolutely continuous, and by Theorem $2\left\{z_{n}\right\}$ is weakly conditionally compact in $L^{\prime}(B, \tau)$. Consequently $f(y)$ is strongly continuons on the unit sphere of $B$, and there exists $x \in L^{\prime}\left(B_{1}, \mu\right)$ such that $f(y)=\mu(x y)$ for all $y \in B$. Let $y^{e}$ be the conditional expectation of $y \in A$ relative to $B_{1}$, then by the same computation of the proof of Theorem 3, we get the equation (6) for $\left\{x_{n}\right\}$ in the place of $\left\{x_{n_{k}}\right\}$.

\section{REFERENCES}

[1] R. G. Bartle, N. Dunford and J. Schivartz, Weak compactness and vector measures. Canadian Journ. Math. 8 (1955), 289-305.

[2] J. Dixmier, Formes linéaires sur un anneau d’opérateurs. Bull. Soc. Math. France $81(1953), 6-39$.

[3] H. A. Dye, The Radon-Nikodym theorem for finite rings of operator. Trans. Amer. Math. Soc. 72 (1952), 234-280.

[4] W.F. Eberlein, Weak compactness in Banach space. Proc. Nat. Acad. Sci. U.S. A. 33 (1947), 51-53.

[5] S. KaKUTANi, Concrete representation of abstract $(L)$-spaces and the mean ergodic theorem. Ann. of Math. 42 (1941), 523-537.

[6] M. Nakamura and T. Turumaru, Expectations in an operator algebra. Tôhoku Math. Journ. 6 (1954), 182-188.

[7] S. SAKs, Addition to the note on some functionals. Trans. Amer. Math. Soc. 35 (1931), 965-970.

[8] I. E. Segal, A non-commutative extension of abstract integration. Ann. of Math. 57 (1953), 401-457.

[9] V. Š́ulian, Über lineare topologische Räume. Recueil Math. 49 (1940).

[10] G. Sunouchi, On the sequence of additive set functions. Journ. Math. Soc. Japan 3 (1951), 290-295.

[11] H. Umegaki, Conditional expectation in an operator algebra. Tôhoku Math. Journ. 6 (1954), $177-181$.

[12] H. Umegaki, Conditional expectation in an operator algebra II. Tôhoku Math. Journ. $8(1956), 86-100$.

Department of Mathematics,

Tokyo Insititute of Technology. 